\title{
Molecular Dynamics Simulations of the Solidification of Pure Aluminium
}

\author{
Michail Papanikolaou*, Konstantinos Salonitis and Mark Jolly \\ Manufacturing Theme, Cranfield University, Cranfield, MK430AL, United Kingdom \\ ${ }^{*}$ Corresponding author: Michail Papanikolaou, m.papanikolaou@cranfield.ac.uk
}

\begin{abstract}
Despite the continuous and remarkable development of experimental techniques for the investigation of microstructures and the growth of nuclei during the solidification of metals, there are still unknown territories around the topic of nucleation during solidification. Such nanoscale phenomena can be effectively observed by means of Molecular Dynamics (MD) simulations which can provide a deep insight into the formation of nuclei and the induced crystal structures. In this study, MD simulations have been performed to investigate the solidification of Aluminium melt and the effects of process parameters such as the cooling rate and hydrostatic pressure on the final properties of the solidified material. A large number of Aluminium atoms have been used in order to investigate the grain growth over time solidification. The population of the Face Centred Cubic (FCC) and amorphous (or non-crystalline) phases has been recorded during the evolution of the process to illustrate the nanoscale mechanisms during solidification. Finally, the exothermic nature of the solidification process has been effectively captured by measuring the temperature of the $\mathrm{Al}$ atoms during grain formation.
\end{abstract}

Keywords: Molecular Dynamics, Solidification, Nucleation, Pressure, Aluminium

\section{Introduction}

In the early 1950s Hall and Petch made the revolutionary observation that the grain size of metallic materials is correlated to their mechanical properties [1,2]. More specifically, 1 they stated that smaller grain sizes lead to higher yield stress and mathematically described the relation between the yield stress and the average grain diameter, which is widely known as the Hall-Petch equation [1]. Later on, researchers expanded the research area around this topic and suggested that the grain size can also be correlated to the ductile-brittle transition, hardness, fatigue and creep behaviour [3]. The reason for the improvement of the mechanical strength for smaller grain sizes is that smaller grains hinder the movement of dislocations while higher external stress needs to be applied for their propagation.

Several ways of improving the grain structure have been reported over the past decades. Three of the most popular ones for reducing the grain size are: (a) the use of alloying elements such as grain refiners [4], (b) controlling the cooling rate [5] and (c) controlling the pressure during casting [6]. More specifically, grain refiners foster heterogeneous nucleation and increase the number of nucleation sites in the melt. For high values of the cooling rate, the melt is quenched to a lower temperature than the equilibrium melting point. This circumstance promotes the formation of additional nuclei in the melt which in turn lead to grain refinement upon solidification [7]. The mechanism of grain refinement under high external pressure can be explained by considering the Clausius-Clapeyron relation which relates the slope of the temperature-pressure equilibrium for coexisting phases, e.g. liquid and solid, to the specific enthalpy for phase change and the specific volume change of the phase transition [8]. After some algebraic manipulation it can be concluded that the critical radius for nucleation decreases for 
higher values of pressure [9]. As a result, nuclei can be quickly formed when pressure is applied and the number of nuclei/grains per unit volume increases.

The real time observation of the nucleation and grain refinement processes is not trivial as extremely high resolution is required. Although the experimental equipment and the adopted measurement techniques have remarkably evolved, there are still challenges associated with the real time observation of the nuclei formation during solidification in the bulk material [10]. Obtaining atomic resolution images from scattering and diffraction experiments is not yet feasible. On the other hand, the Classical Nucleation Theory (CNT) [11] frequently fails to accurately predict experimental nucleation rates [12] as it is based on the assumption that nucleation can occur with equal probability at any point within the melt, which does not hold in reality [13].

The aforementioned shortcomings can be addressed via the Molecular Dynamics (MD) simulation technique. This computer simulation method provides high resolution information while no ab initio assumptions are required for the physical system under examination. The first MD simulations on this topic considered quite small atom populations and were limited to two dimensions [14]. However, as suggested by Streitz et al. [15], in order to reproduce realistic and size-independent solidified structures via means of MD simulations large atom populations should be considered. The evolution of the computational power and the development of high-performance computing clusters has allowed for large scale MD simulations of solidification. Large MD simulations have significantly contributed to the in-depth understanding of the phenomena taking place during solidification and the process parameters affecting the structural properties of the solidified structure. Some of the topics investigated include the effect of the cooling rate on the solidified structure [16], the structural and dynamical properties of rapidly quenched metals [17], nucleation and grain growth [18]. The largest MD simulation ever reported has been performed by Shibuta et al. [19] who investigated homogeneous nucleation from an undercooled iron melt containing 1 billion atoms. Based on their observations, the authors proposed a novel nucleation model, which is based in heterogeneity. In contrast to the classical nucleation models (spontaneous and sporadic) they found that small satellite-like grains are formed in the vicinity of earlier formed grains.

Considering the challenges encountered during the real-time experimental observation of the nucleation process during solidification, it is clear that MD simulations offer some significant advantages compared to other computational methods and experimental techniques, which lack atomic resolution and make ab initio assumptions. In this study, MD simulations have been employed to investigate the effects of the cooling rate and pressure on the properties of solidified aluminium. The obtained results suggest that the cooling rate significantly affects the grain nucleation and growth as well as the grain distribution in the solidified structure. Therefore its value should be thoughtfully controlled in order to obtain the desired material properties. On the other hand, pressure increases the melting point, increases the growth rate but does not significantly alter the final average grain size.

\section{Methodology}

The simulation setup consists of a simulation box with periodic boundary conditions containing $1,000,188$ Aluminium (Al) atoms. The Al atoms are initially arranged in a FCC lattice with a lattice constant equal to $4.046 \AA$. The Finnis-Sinclair (FS) potential [20] has been employed to model the interatomic interactions between the $\mathrm{Al}$ atoms. The FS potential has been extensively used to investigate nucleation and solidification via MD simulations $[19,21,22]$. The FS potential takes into account the local density dependence of the interatomic forces by considering a repulsive term and an attractive term (square root) which is dependent on the forces exerted on a specific atom by its neighbours:

$$
E_{\text {tot }}=\frac{1}{2} \sum_{i=1}^{N} \sum_{j=1}^{N} V_{i j}\left(r_{i j}\right)-A \sum_{i=1}^{N} \sqrt{\rho_{i}}
$$


where $V_{i j}$ is the potential energy for the atom pair $i j, r_{i j}$ the distance between the atoms $i$ and $j, A$ is the binding energy and $\rho_{i}$ the local electronic charge in the vicinity of atom $i$.

The Al melt was prepared by isothermally heating the Al crystal up to $1173 \mathrm{~K}$ using the isothermalisobaric ensemble (NPT) ensemble. The melt was subsequently equilibrated for 20,000 timesteps; at the end of the equilibration process there were no FCC atoms left in the domain. Following the equilibration stage, the melt was quenched under three cooling rates (1, 2 and $4 \mathrm{~K} / \mathrm{ps})$ and three values of pressure $(0,0.5$ and $1 \mathrm{GPa})$, i.e., 9 simulations were performed in total. Following the quenching process, the simulation domain was further equilibrated for 100,000 timesteps ( $2 \mathrm{~ns}$ ) at $273 \mathrm{~K}$ in order to allow for some additional time for the FCC grains to grow and obtain the final solidified structure. The values of the simulation parameters are summarised in Table 1.

Table 1: Simulation parameters

\begin{tabular}{|l|l|}
\hline Number of atoms & $1,000,188$ \\
\hline Material & Aluminium \\
\hline Ensemble & NPT \\
\hline Potential & Finnis-Sinclair (FS) \\
\hline Timestep (fs) & 2 \\
\hline Pressure $(\mathbf{G P a})$ & $0,0.5$ and 1 \\
\hline Cooling rate (K/ps) & 1,2 and 4 \\
\hline
\end{tabular}

In this study, the per-atom temperature was evaluated in order to visualise the temperature distribution over the simulation domain. The per atom temperature has been measured using the equipartition theorem [23] as stated below:

$$
T=\frac{K E}{\frac{\operatorname{dim}}{2} N k_{B}}
$$

where $N$ is number of atoms, $k_{B}=1.38 \cdot 10^{-23} \mathrm{~m}^{2} \mathrm{~kg} \mathrm{~s}^{-2} \mathrm{~K}^{-1}$ the Boltzmann constant and $\operatorname{dim}=3$ the number of dimensions of the simulation. The kinetic energy $(K E)$ is given by:

$$
K E=\frac{1}{2} \sum_{i=1}^{N} m_{i} v_{i}^{2}
$$

where $m_{i}$ and $v_{i}$ are the mass and velocity of the atom $i$ at a specific timestep.

With regard to structural properties, the Radial Distribution Function (RDF) has been employed as a metric of the local ordering of atoms. The RDF has been calculated as follows:

$$
g(r)=\frac{V}{N^{2}}\left\langle\frac{N(r)}{4 \pi r^{2} \Delta r}\right\rangle
$$

where $V$ is the system volume, $N$ the population of the simulation atoms, and $N(r)$ the number of atoms lying within the spherical shell $r-\Delta r / 2<r_{i}<r+\Delta r / 2$.

The open source LAMMPS Molecular Dynamics Simulator [24] has been used to perform the large scale MD simulations. Each simulation was performed on 32 cores of the Delta HPC facility of the Cranfield University while the corresponding running time ranged between 6 and 24 hours. The OVITO open source software [25] along with its integrated modifiers and some in house post processing scripts were utilised for the post-processing and visualisation of the results.

\section{Results \& Discussion}

As mentioned in the previous section, the prepared $\mathrm{Al}$ melt was quenched under 3 different cooling rates $(1,2$ and $4 \mathrm{~K} / \mathrm{ps})$. The effect of the cooling rate on the solidified structure is illustrated in Figure 1. 
It is evident that the cooling rate significantly affects the number of grains in the final structure. More specifically, additional grains are formed during rapid quenching; this is because solidification occurs in a wider range of temperatures compared to lower cooling rates and additional nucleation points are generated. This result is in agreement with previous investigations [16].

(a)

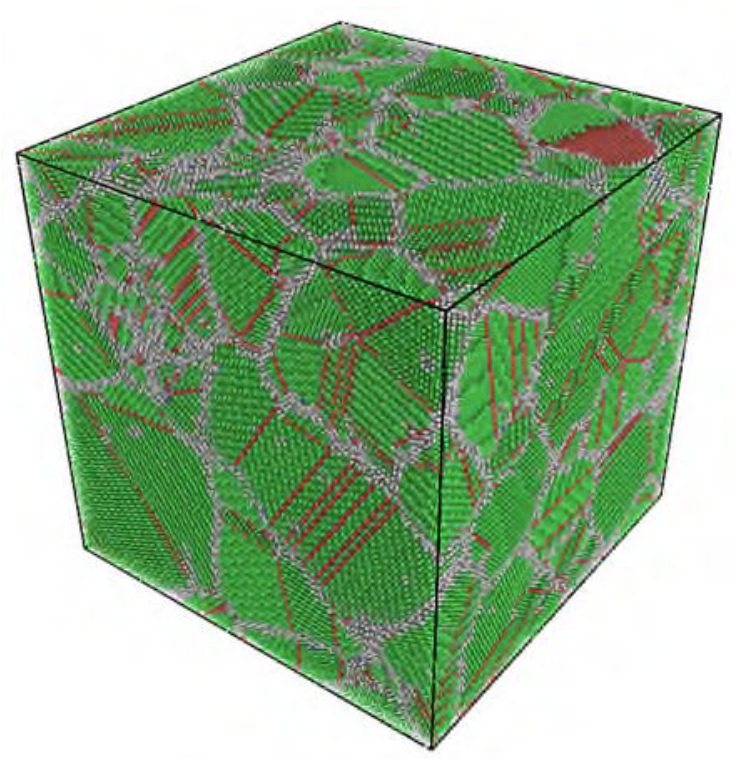

(b)

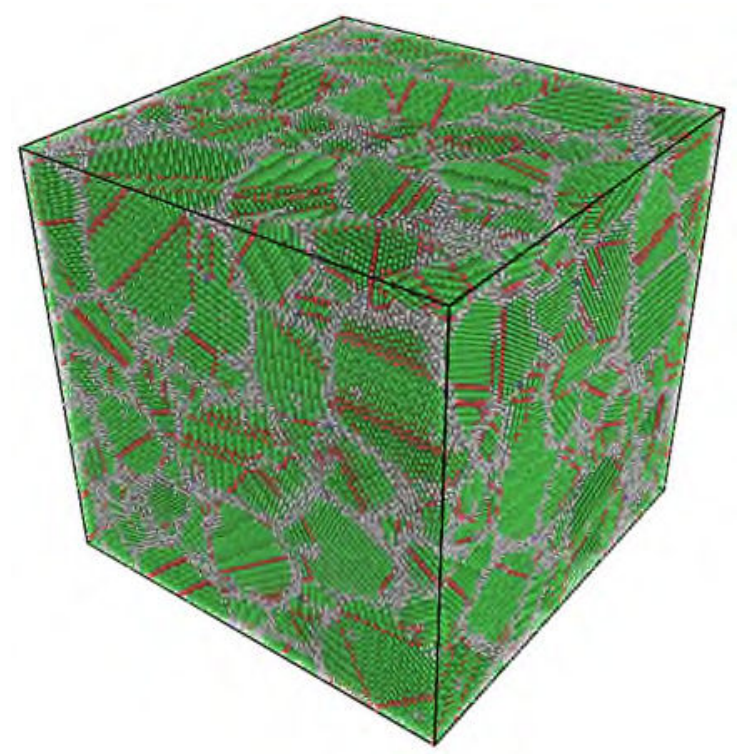

(c)

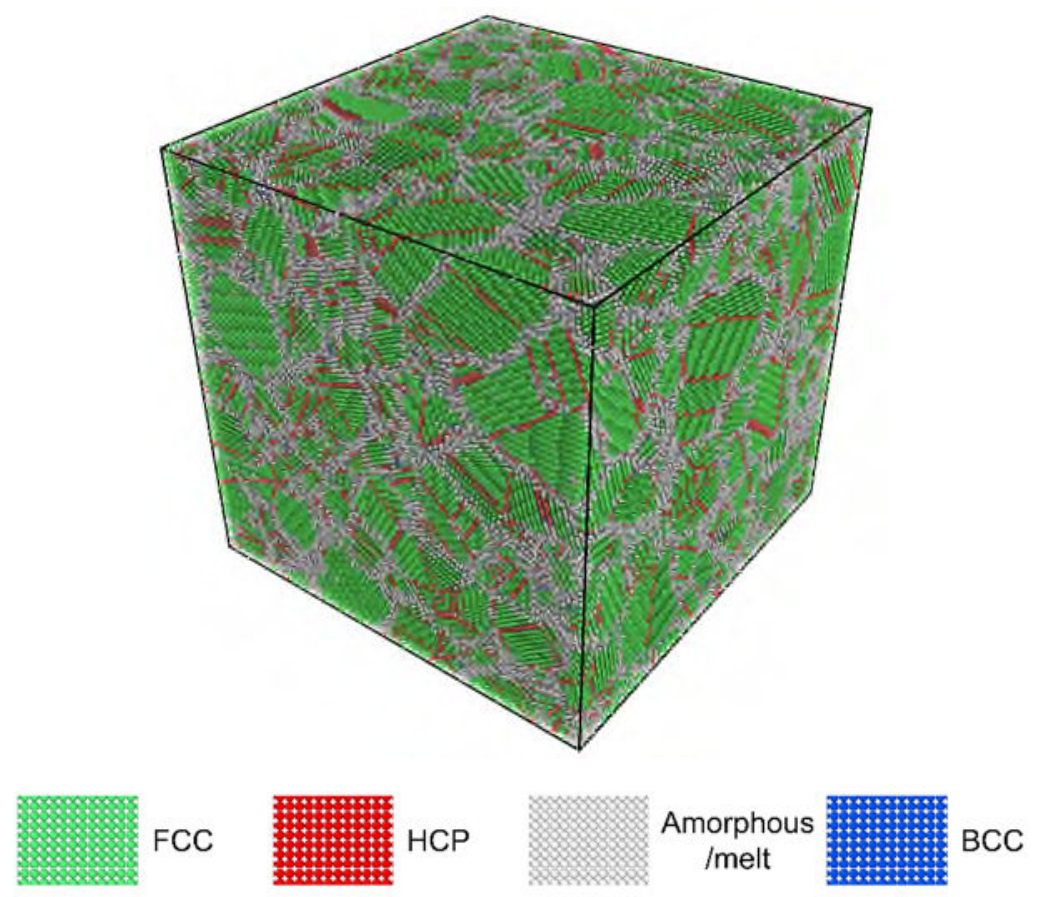

Figure 1: Solidified structure for a cooling rate of (a) $1 \mathrm{~K} / \mathrm{ps}$, (b) $2 \mathrm{~K} / \mathrm{ps}$ and (c) $4 \mathrm{~K} / \mathrm{ps}$

In order to visualise the nucleation process, the cluster analysis modifier of the OVITO software [25] was employed. Clusters were identified via a distance based neighbour criterion, according to which two atoms separated by a distance smaller than a specified value belong to the same cluster or grain. This critical value was set equal to the lattice constant of Aluminium (4.046 $)$. The grain formation for a cooling rate of $1 \mathrm{~K} / \mathrm{ps}$ is shown in Figure 2. It can be observed that grains grow in a sphere-like manner while the atoms of the liquid/melt phase get attached to the potential FCC sites of the grain boundary interface. 
(a)

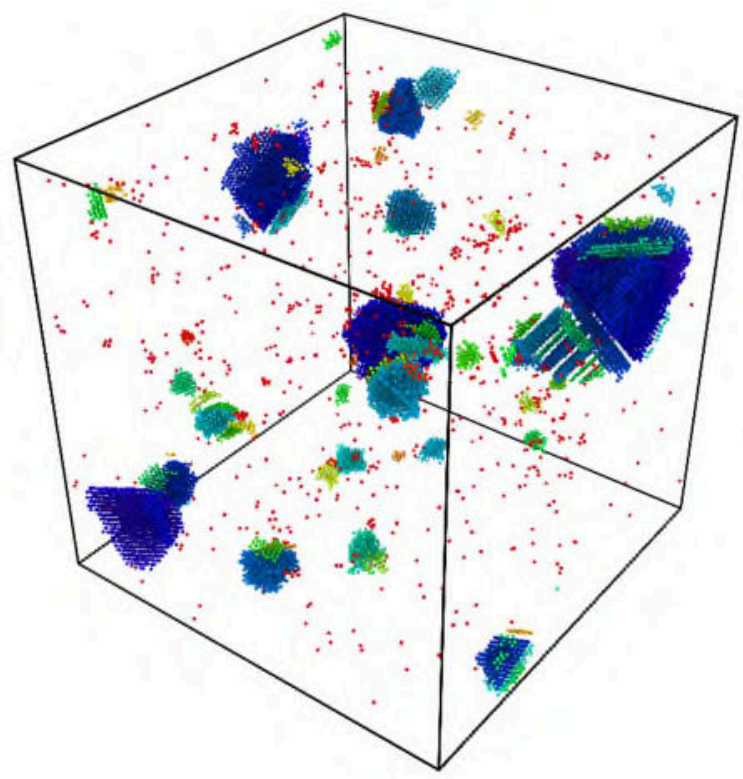

(c)

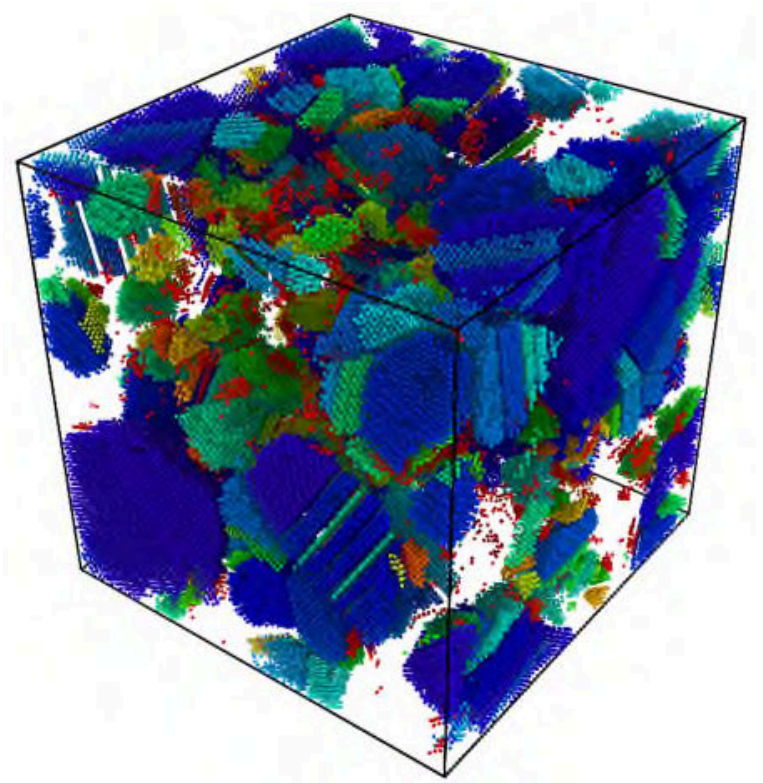

(b)

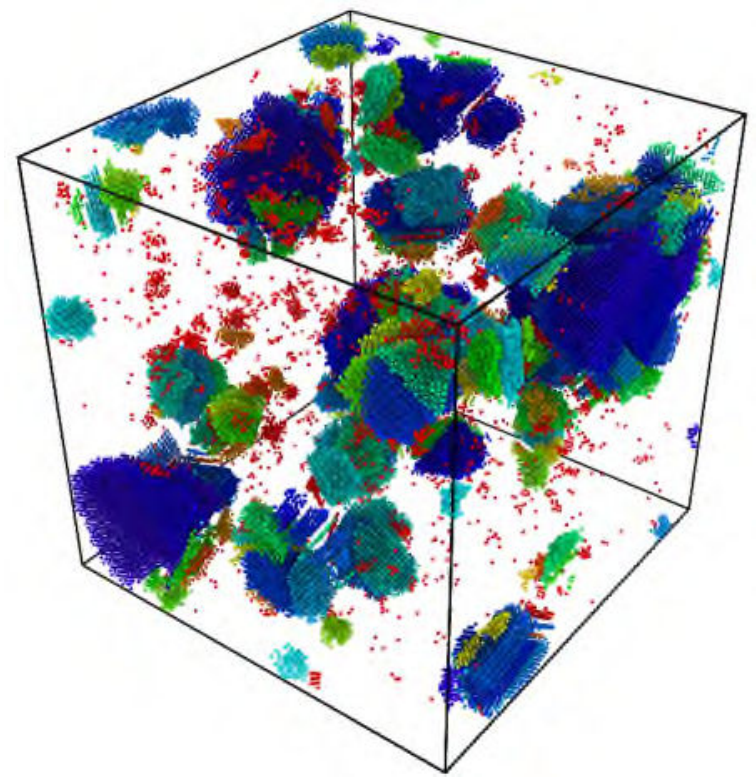

(d)

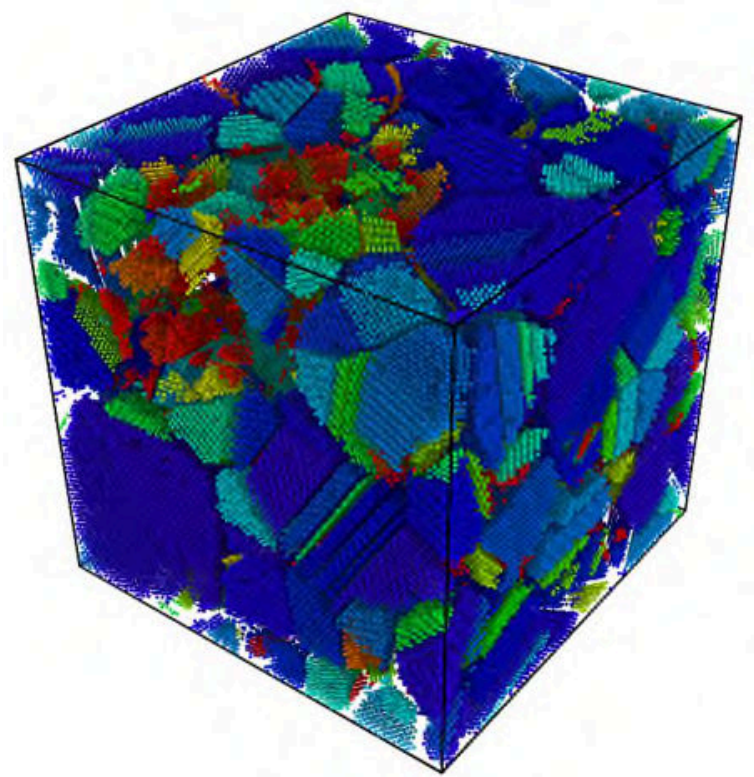

Figure 2: Nucleation for a cooling rate of $1 \mathrm{~K} / \mathrm{ps}$ at (a) $543 \mathrm{~K}$, (b) $525 \mathrm{~K}$, (c) $507 \mathrm{~K}$ and (d) $489 \mathrm{~K}$.

Nucleation and solidification are accompanied by the release of the latent heat of crystallisation and a steep reduction of the potential energy; this is because the FCC crystal phase is energetically favourable. The effects of pressure and cooling rate on the potential energy have been investigated. As shown in Figure 3, the potential energy drops linearly with temperature for all cooling rates under examination. However, lower cooling rates contribute to a steeper reduction of the potential energy when solidification commences, i.e. solidification occurs over a shorter range of temperatures. As expected, the magnitude of the potential energy of the system increases with pressure. This is more pronounced when the $\mathrm{Al}$ atoms are in the melt phase. Finally, it can be observed that solidification commences at higher temperatures when pressure is high; this is in agreement with previous investigations which suggest that the melting point of metals strongly depends on the static pressure [26]. 
(a)

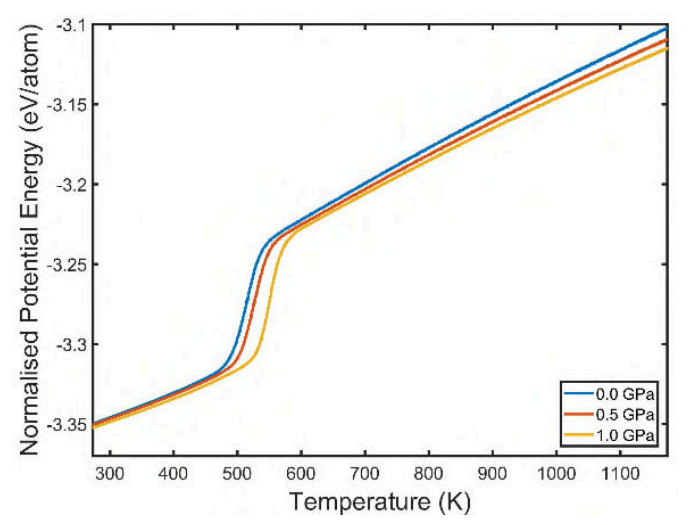

(b)

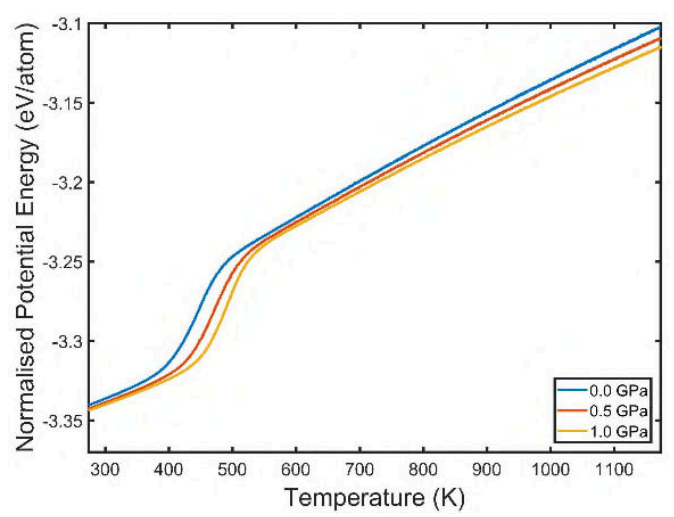

(c)

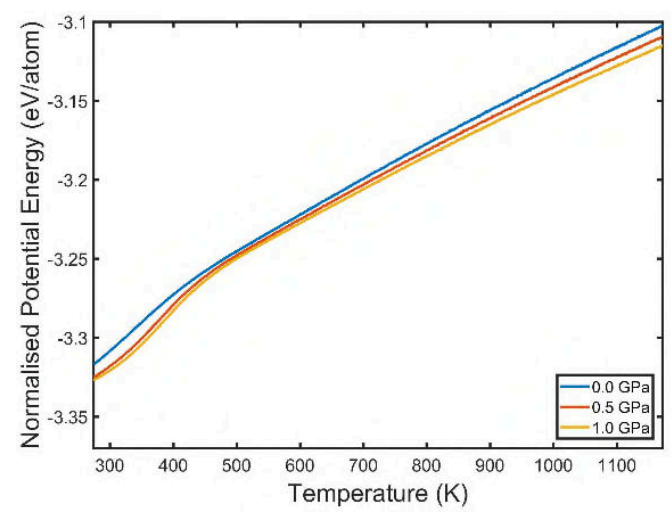

Figure 3: Normalised potential energy vs. temperature for a cooling rate of (a) $1 \mathrm{~K} / \mathrm{ps}$, (b) $2 \mathrm{~K} / \mathrm{ps}$ and (c) $4 \mathrm{~K} / \mathrm{ps}$

\section{Common Neighbour Analysis}

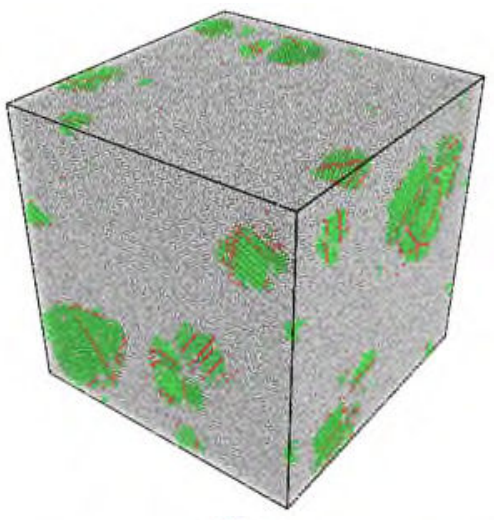

(a)

(b)

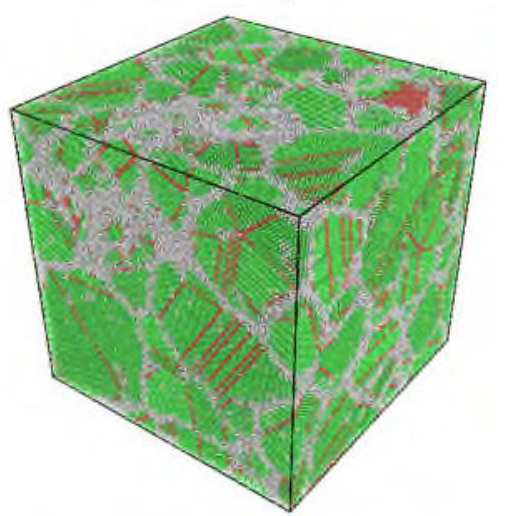

Potential Energy Distribution
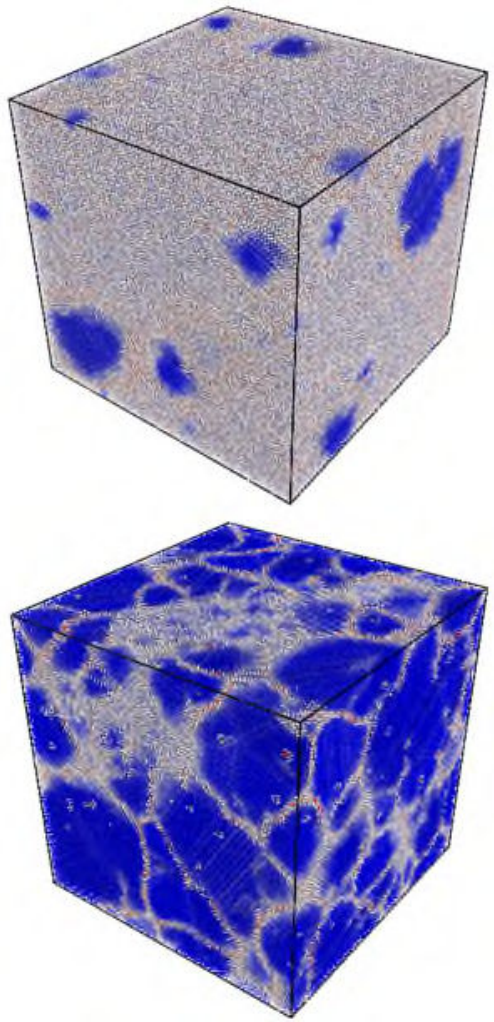

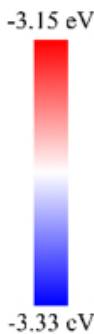

Figure 4: Common Neighbour Analysis and potential energy distribution for a cooling rate of $1 \mathrm{~K} / \mathrm{ps}(\mathrm{a}) \mathrm{T}=543 \mathrm{~K}$ and $(\mathrm{b})$ $T=489 \mathrm{~K}$ 
Figure 3 provides a quantitative estimate of the potential energy evolution during the process. However, in order to obtain a clearer understanding of the local potential energy evolution during the solidification process, the per atom potential energy was calculated and averaged over time windows of $N_{t s} / 50$ timesteps. $N_{t s}$ is the total number of timesteps of the quenching process, being dependent on the cooling rate. In Figure 4, the results of the Common Neighbour Analysis (CNA) and the local potential energy are being compared for identical temperature values. As expected, the potential energy is not uniformly distributed over the simulation domain but lower values (blue colour) can be observed at the areas occupied by grains, where the energetically favourable FCC and HCP phases are dominant. For the identical reason, the potential energy of the atoms at the grain boundaries or the ones corresponding to the liquid phase, which are non-crystalline, is higher.

(a)

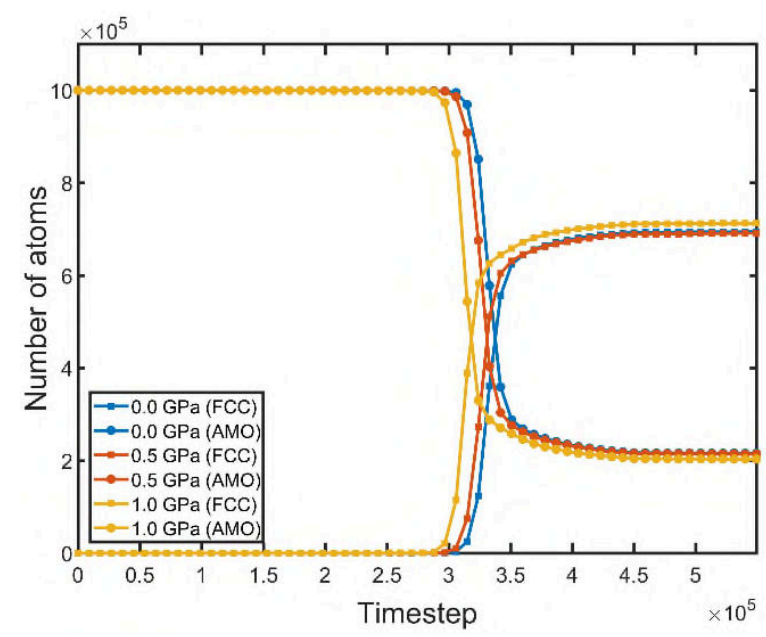

(b)

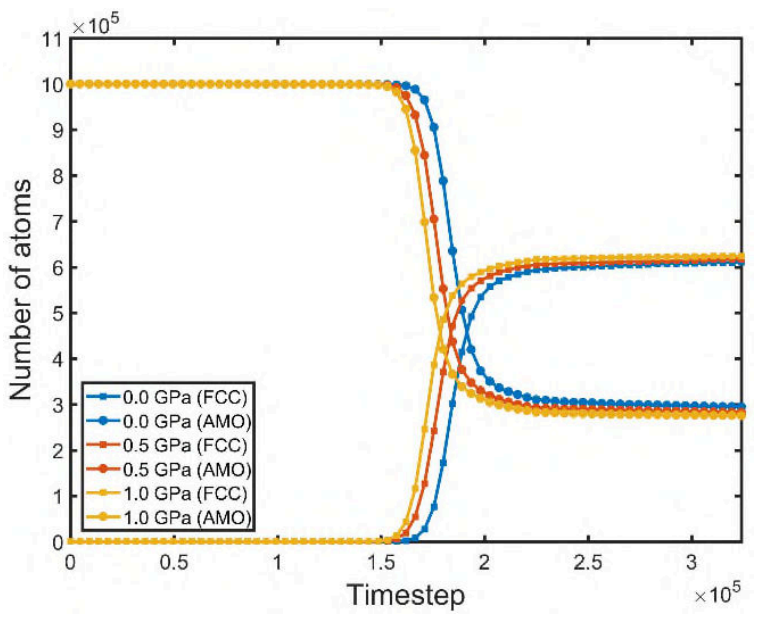

(c)

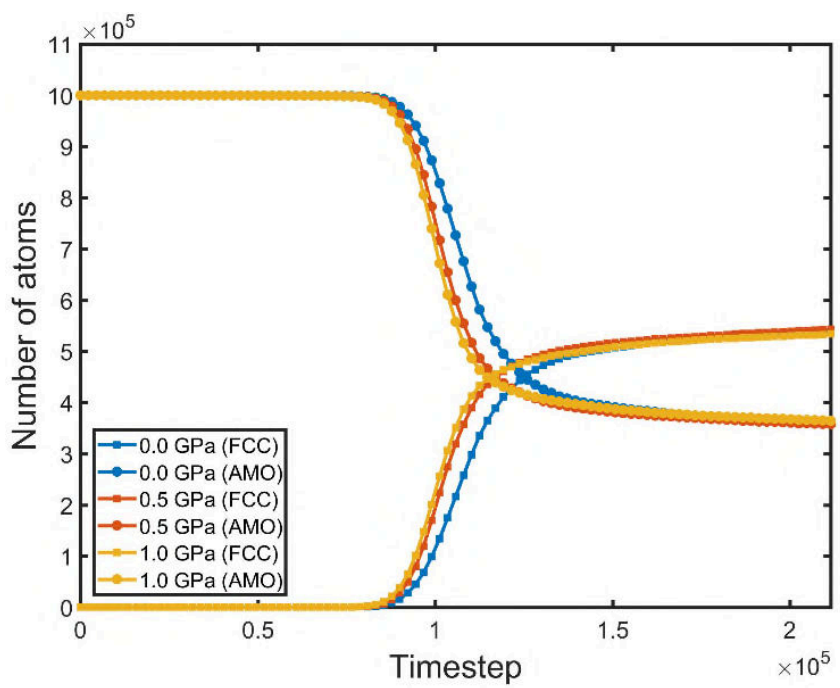

Figure 5: Evolution of Face Centred Cubic (FCC) and amorphous (AMO) populations over time for a cooling rate of (a) 1 $K / p s$, (b) $2 \mathrm{~K} / \mathrm{ps}$ and (c) $4 \mathrm{~K} / \mathrm{ps}$

In Figure 5 the populations of the Face Centred Cubic (FCC) and amorphous (AMO) structures have been plotted over time. As expected, the population of the amorphous structures is initially (Timestep $=0$ ) equal to the total number of atoms $\left(10^{6}\right)$, because $\mathrm{Al}$ is in the molten state. As the simulation temperature drops, the population of the amorphous phase undergoes a steep decay. This abrupt change corresponds to the point in time when the first nuclei (grains) are generated. At the same timestep and for the same reason, the population of the FCC phase starts to sharply rise. Towards the end of the quenching and equilibration processes, the populations of both phases asymptotically reach a constant 
value. It has to be mentioned that high cooling rates lead to high populated FCC phase and consequently to low populated amorphous phase. This is because of two main reasons: (a) high cooling rates lead to the rapid immobilisation of atoms while there is no sufficient time to obtain the favourable FCC/HCP structure and (b) high cooling rates lead to the formation of a high number of grains resulting in increased area of grain boundaries and additional atoms being in amorphous state.

(a)

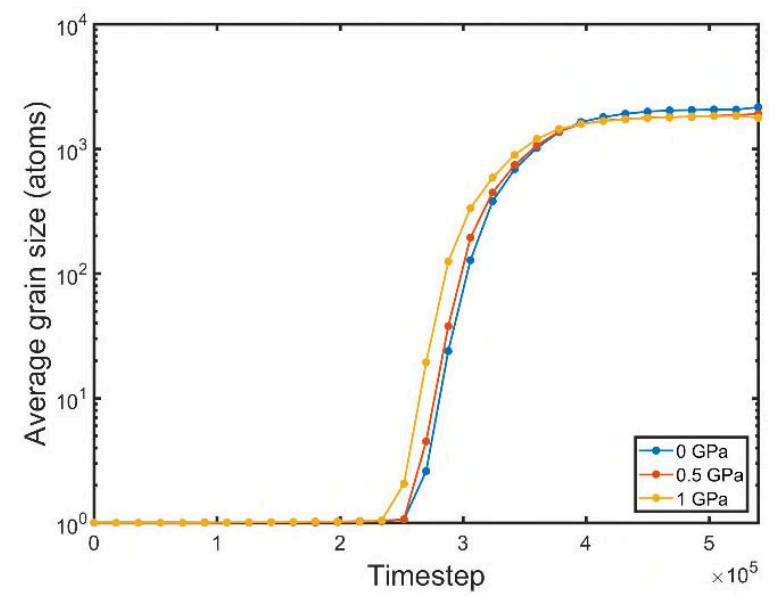

(b)

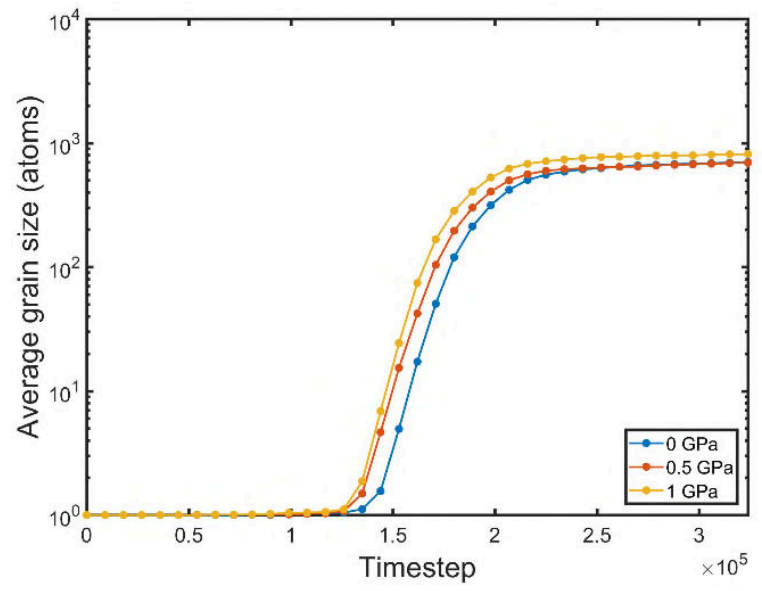

(c)

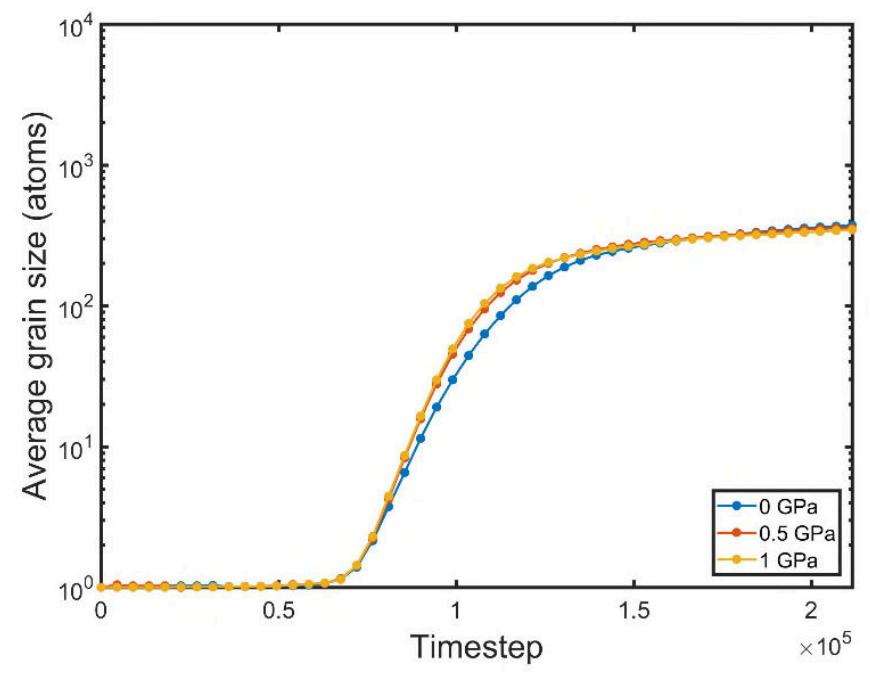

Figure 6: Average grain size for a cooling rate of (a) $1 \mathrm{~K} / \mathrm{ps}$, (b) $2 \mathrm{~K} / \mathrm{ps}$ and (c) $4 \mathrm{~K} / \mathrm{ps}$

As stated in the introduction, a large variety of mechanical properties of metals depend on the average grain size. In Figure 6 the average grain size is plotted as a function of the timestep for various cooling rates $(1,2,4 \mathrm{~K} / \mathrm{ps})$. During quenching, the average grain size starts sharply increasing when the first grains are formed. This sharp increase is followed by a linear and an asymptotic one, as the grains obtain their final size during equilibration. In Figure 6 it can be observed that the cooling rate plays a significant role on the average grain size. For the lowest cooling rate $(1 \mathrm{~K} / \mathrm{ps})$, the average grain size is about equal to 1000 atoms. The corresponding equivalent diameters $\left(d_{g}\right)$ were calculated (assuming spherical grains) as follows:

$$
d_{g}=2 \cdot \sqrt[3]{\frac{N m}{\frac{4}{3} \pi \rho}}
$$


where $N$ is the number of the grain atoms, $m=4.48 \cdot 10^{-26} \mathrm{~kg}$ the mass of an $\mathrm{Al}$ atom and $\rho=$ $2710 \mathrm{~kg} / \mathrm{m}^{3}$ the Aluminium density. On the other hand, although pressure increases the melting point and boosts nucleation it does not appear to significantly affect the final grain size.

The final part of this investigation is focused on measuring the local temperature over the simulation domain and exploring the exothermic nature of the nucleation process. The local (per atom) temperature has been measured using equations (2) and (3). Similarly to the estimation of the local potential energy, in order to obtain an accurate measurement, the temperature of each atom was calculated and averaged over time windows of $N_{t s} / 50$ timesteps, where $N_{t s}$ is the total number of timesteps of the quenching process, being dependent on the cooling rate. In Figure 7 the results of the Common Neighbour Analysis are being compared to the corresponding temperature profiles when the average domain temperature is equal to (a) $525 \mathrm{~K}$ and (b) $507 \mathrm{~K}$. It is apparent that the temperature in the vicinity of the formed grains is much higher compared to the surrounding melt. The elevated temperature at the region of the grains is a result of the release of the latent heat of crystallisation. As quenching proceeds (Figure 7(b)), it can be observed that the temperature of the simulation domain (melt and grains) drops while the more recently formed grains can still be spotted due to their elevated temperature.

(a)

Common Neighbour Analysis

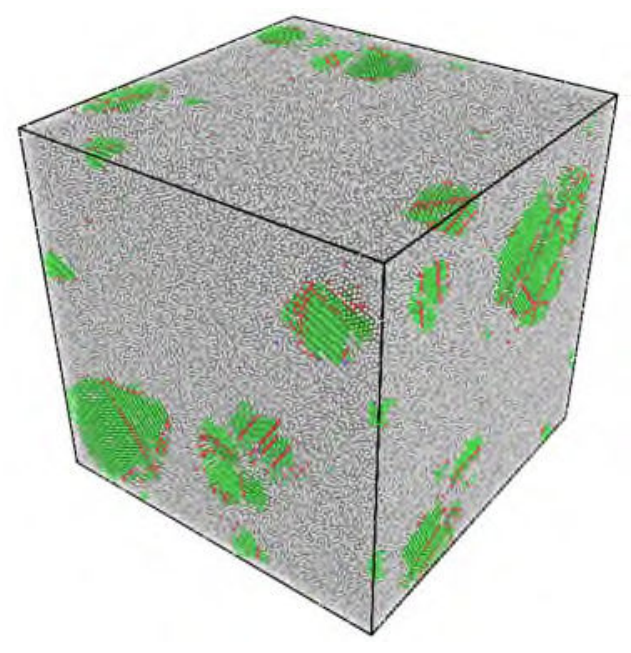

(b)

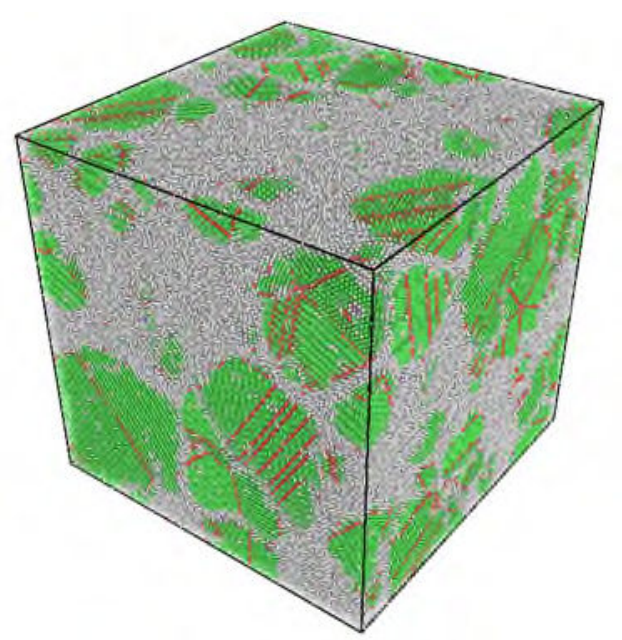

Temperature distribution

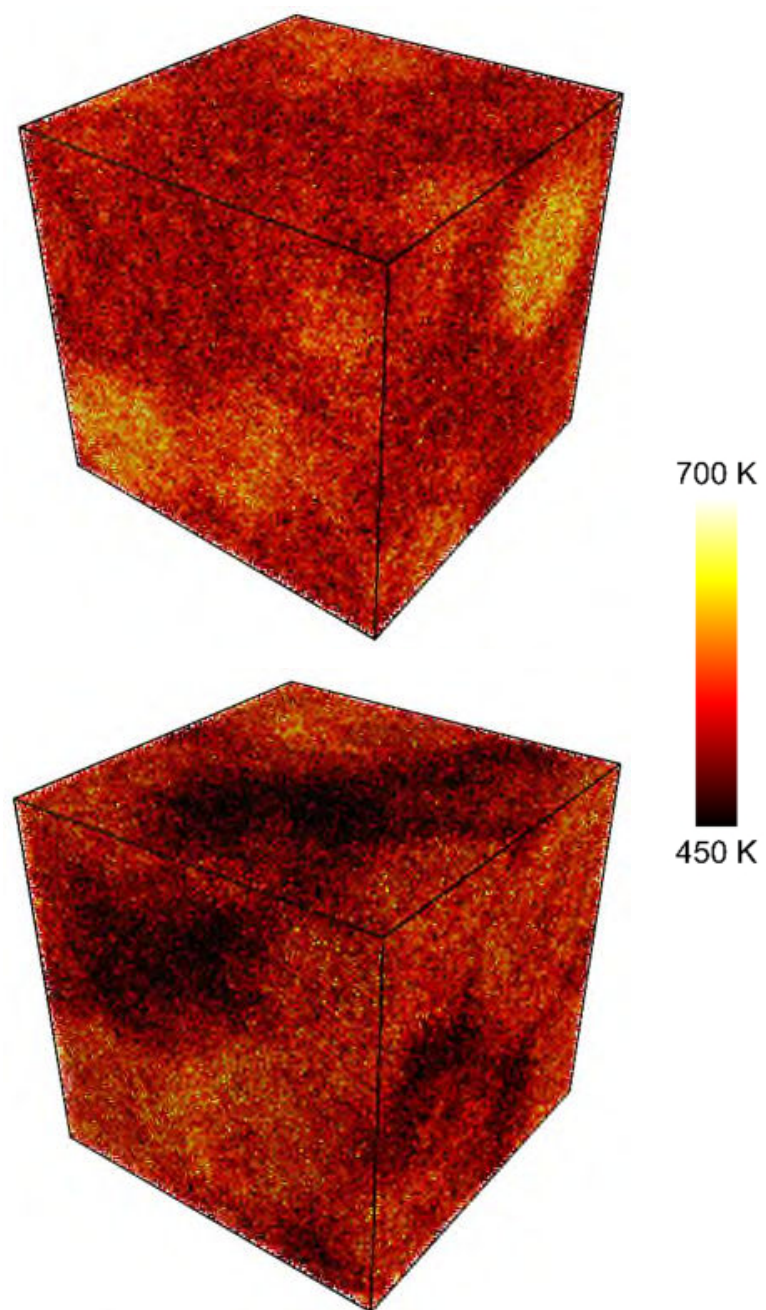

Figure 7: Common Neighbour Analysis and local temperature distribution for a cooling rate of $1 \mathrm{~K} / \mathrm{ps}$ at (a) $T=525 \mathrm{~K}$ and (b) $T=507 \mathrm{~K}$ 


\section{Conclusions}

In this study, Molecular Dynamics (MD) simulations have been employed to investigate the effects of both the cooling rate and the applied pressure on the solidification of pure Aluminium (Al). The interactions between the $\mathrm{Al}$ atoms were modelled using the Finnis-Sinclair (FS) potential. A simulation domain containing 1 million $\mathrm{Al}$ atoms arranged in a FCC lattice was initially heated up to $1173 \mathrm{~K}$ and equilibrated. Subsequently, the prepared $\mathrm{Al}$ melt was quenched to $273 \mathrm{~K}$ under various cooling rates (1, 2 and $4 \mathrm{~K} / \mathrm{ps})$ and values of pressure $(0,0.5$ and $1 \mathrm{GPa})$. Quenching was followed by a final equilibration stage in order to allow the simulation domain to obtain its final solidified structure. The main conclusions drawn from this study are summarised below:

- In this investigation, the exothermic nature of the solidification process (release of the latent heat of crystallisation) has been effectively captured via MD simulations, which do not make any ab initio assumptions (unlike CFD or coarse grained models). According to the results, solidification occurs in a wider range of temperatures when the cooling rate is high.

- MD offers the advantage of atomic resolution, which allows for the real time observation of the solidification/nucleation kinetics and dynamics. The grain size has been found to be dependent on the cooling rate. More specifically, high cooling rates contribute towards the formation of a large number of small grains while low cooling rates lead to fewer but larger ones.

- This is the first investigation on the effects of pressure on the solidification of pure Al via means of MD. The obtained results suggest that although high pressure boosts nucleation and increases the melting point, the average grain size appears to be independent of the applied pressure.

\section{Acknowledgements}

The authors would like to acknowledge the UK EPSRC project "Energy Resilient Manufacturing 2: Small Is Beautiful Phase 2 (SIB2)" for funding this work under grant EP/P012272/1. 


\section{References}

[1] E.O. Hall, The Deformation and Ageing of Mild Steel: III Discussion of Results, Proc. Phys. Soc. Sect. B. 64 (1951) 747-753. doi:10.1088/0370-1301/64/9/303.

[2] N.J. Petch, The cleavage strength of polycrystals, J. Iron Steel Inst. 174 (1953) 25-28.

[3] R.W. Armstrong, The influence of polycrystal grain size on several mechanical properties of materials, Metall. Mater. Trans. B. 1 (1970) 1169-1176. doi:10.1007/BF02900227.

[4] K.T. Kashyap, T. Chandrashekar, Effects and mechanisms of grain refinement in aluminium alloys, Bull. Mater. Sci. 24 (2001) 345-353.

[5] M.A. Easton, D.H. StJohn, Improved prediction of the grain size of aluminum alloys that includes the effect of cooling rate, Mater. Sci. Eng. A. 486 (2008) 8-13. doi:10.1016/J.MSEA.2007.11.009.

[6] P. Ma, Z.J. Wei, Y.D. Jia, C.M. Zou, S. Scudino, K.G. Prashanth, Z.S. Yu, S.L. Yang, C.G. Li, J. Eckert, Effect of high pressure solidification on tensile properties and strengthening mechanisms of Al-20Si, J. Alloys Compd. 688 (2016) 88-93. doi:10.1016/J.JALLCOM.2016.07.016.

[7] S. Shabestari, M. Malekan, Thermal analysis study of the effect of the cooling rate on the microstructure and solidification parameters of 319 aluminum alloy, Can. Metall. Q. 44 (2005) 305-312. doi:10.1179/cmq.2005.44.3.305.

[8] Y.A. Cengel, M.A. Boles, Thermodynamics: an engineering approach, Sea. 1000 (2002) 8862.

[9] J.J. Sobczak, L. Drenchev, R. Asthana, Effect of pressure on solidification of metallic materials, Int. J. Cast Met. Res. 25 (2012) 1-14.

[10] N. Iqbal, N.H. van Dijk, S.E. Offerman, M.P. Moret, L. Katgerman, G.J. Kearley, Real-time observation of grain nucleation and growth during solidification of aluminium alloys, Acta Mater. 53 (2005) 28752880. doi:10.1016/J.ACTAMAT.2005.02.045.

[11] M. Volmer, A. Weber, Keimbildung in übersättigten Gebilden, Zeitschrift Für Phys. Chemie. 119 (1926) 277-301.

[12] E.D. Zanotto, P.F. James, Experimental tests of the classical nucleation theory for glasses, J. Non. Cryst. Solids. 74 (1985) 373-394.

[13] J. Russo, H. Tanaka, The microscopic pathway to crystallization in supercooled liquids, Sci. Rep. 2 (2012) 505.

[14] F. Celestini, J.-M. Debierre, Nonequilibrium molecular dynamics simulation of rapid directional solidification, Phys. Rev. B. 62 (2000) 14006-14011. doi:10.1103/PhysRevB.62.14006.

[15] F.H. Streitz, J.N. Glosli, M. V Patel, Beyond finite-size scaling in solidification simulations, Phys. Rev. Lett. 96 (2006) 225701.

[16] Z.Y. Hou, K.J. Dong, Z.A. Tian, R.S. Liu, Z. Wang, J.G. Wang, Cooling rate dependence of solidification for liquid aluminium: a large-scale molecular dynamics simulation study, Phys. Chem. Chem. Phys. 18 (2016) 17461-17469. doi:10.1039/C6CP02172G.

[17] B. Shen, C.Y. Liu, Y. Jia, G.Q. Yue, F.S. Ke, H.B. Zhao, L.Y. Chen, S.Y. Wang, C.Z. Wang, K.M. Ho, Molecular dynamics simulation studies of structural and dynamical properties of rapidly quenched $\mathrm{Al}, \mathrm{J}$. Non. Cryst. Solids. 383 (2014) 13-20.

[18] A. Mahata, M.A. Zaeem, M.I. Baskes, Understanding homogeneous nucleation in solidification of aluminum by molecular dynamics simulations, Model. Simul. Mater. Sci. Eng. 26 (2018) 025007. doi:10.1088/1361-651X/aa9f36.

[19] Y. Shibuta, S. Sakane, E. Miyoshi, S. Okita, T. Takaki, M. Ohno, Heterogeneity in homogeneous nucleation from billion-atom molecular dynamics simulation of solidification of pure metal, Nat. Commun. 8 (2017) 10. doi:10.1038/s41467-017-00017-5.

[20] M.W. Finnis, J.E. Sinclair, A simple empirical N-body potential for transition metals, Philos. Mag. A. 50 (1984) 45-55.

[21] Y. Watanabe, Y. Shibuta, T. Suzuki, A molecular dynamics study of thermodynamic and kinetic properties of solid--liquid interface for bcc iron, ISIJ Int. 50 (2010) 1158-1164. 
[22] J. Liu, R.L. Davidchack, H.B. Dong, Molecular dynamics calculation of solid-liquid interfacial free energy and its anisotropy during iron solidification, Comput. Mater. Sci. 74 (2013) 92-100. doi:10.1016/J.COMMATSCI.2013.03.018.

[23] K. Huang, Statistical mechanics, Wiley, $1987 . \quad$ https://www.wiley.com/engb/Statistical+Mechanics,+2nd+Edition-p-9780471815181 (accessed August 14, 2019).

[24] LAMMPS Molecular Dynamics Simulator, (n.d.). https://lammps.sandia.gov/.

[25] A. Stukowski, Ovito Open Visualization Tool, (2015).

[26] H. Schlosser, P. Vinet, J. Ferrante, Pressure dependence of the melting temperature of metals, Phys. Rev. B. 40 (1989) 5929-5935. doi:10.1103/PhysRevB.40.5929. 
2020-01-28

\title{
Molecular dynamics simulations of the solidification of pure aluminium
}

\author{
Papanikolaou, Michail
}

Springer

Papanikolaou M, Salonitis K, Jolly M. (2020) Molecular dynamics simulations of the solidification of pure aluminium. In: Light metals 2020, Cham: Springer. pp.158-167

https://doi.org/10.1007/978-3-030-36408-3_22

Downloaded from Cranfield Library Services E-Repository 